\title{
A kinematic theory of rapid human movements
}

\section{Part I. Movement representation and generation}

\section{Réjean Plamondon}

École Polytechnique de Montréal, Département de Génie Électrique et de Génie Informatique, C.P. 6079, Succursale Centre-Ville, Montréal, Canada H3C 3A7

Biol. Cybern. 72, $295-307$ (1995)

Due to an unfortunate typesetting oversight, there was a spelling error in the caption of Figure 7. It should have read J. Blouin instead of J. Bolvin.

\section{A kinematic theory of rapid human movements}

\section{Part II. Movement time and control}

\section{Réjean Plamondon}

École Polytechnique de Montréal, Département de Génie Électrique et de Génie Informatique, C.P. 6079, Succursale Centre-Ville, Montréal, Canada H3C 3A7

Biol. Cybern. 72, 309-320 (1995)

Due to an unfortunate typesetting oversight, equations (3) and (4) were not corrected as indicated in the proof; they should read as follows:

$a_{2}=\frac{\mu_{2} \sigma_{1}^{2}-\mu_{1} \sigma_{2}^{2}}{\sigma_{1}^{2} \sigma_{2}^{2}}$

$a_{3}=\frac{\sigma_{2}^{2} \mu_{1}^{2}-\sigma_{1}^{2} \mu_{2}^{2}+2 \sigma_{1}^{2} \sigma_{2}^{2} \ln \left(\frac{\sigma_{1}}{\sigma_{2}}\right)}{2 \sigma_{1}^{2} \sigma_{2}^{2}}$ 